power London National causes stronger interaction than London Regional. Since their waves are respectively 11 and 45 per cent longer than the local gyro wave-length 235 metres, this result strongly supports the conclusion that resonance exists.

In the experiments with Lille, London National and London Regional, it was usually found that the intensity of the impressed modulation decreased as the modulation frequency increased. This is in accordance with the theory. But on several occasions the intensity with a modulation frequency of 1,000 cycles/sec. was observed to be much stronger than that with a lower frequency: an interesting anomaly in the theory in its present state.

A more complete account of the theory will be given in the issue of the Philosophical Magazine of May.

The Queen's College,

V. A. BAILEY.

Oxford.

April 3.

Nature, 139, 68 (Jan. 9, 1937).

\section{Cosmic Rays and the Magnetic Moment of the Sun}

Ir is generally assumed that the constant intensity of cosmic radiation at sea-level beyond a certain latitude is accounted for by the 'clocking effect' of the atmosphere, which absorbs all particles of energy below the limit of that latitude resulting from the action of the earth's magnetic field. An important consequence of this assumption is that the latitude at which the intensity becomes constant should be displaced towards the geomagnetic poles as the altitude above sea-level increases, since then the thickness of the absorbing air layer decreases and therefore also the energy absorbed. Compton's mountain experiments ${ }^{1}$ already throw doubt on the existence of this displacement, and this doubt is strengthened by Cosyns's stratospheric measurements ${ }^{2}$ which, while admittedly inconclusive, indicate that the intensity remains constant north of $49^{\circ}$ even at the highest altitudes reached.

There are clearly two possible interpretations of this remarkable result. Either the spectrum of primary cosmic radiation has a sharp threshold at the energy corresponding to the geomagnetic threshold at that latitude where the intensity becomes constant, or else, as suggested by Janossy ${ }^{3}$, particles of this energy (and less) are prevented from reaching the earth by the action of the sun's permanent magnetic field, although the moon might also be suspected.

The theory of the allowed cone of cosmic radiation ${ }^{4}$ gives for the geomagnetic threshold at $50^{\circ}$ about 200 millistörmers (mS.) for particles coming horizontally and $300 \mathrm{mS}$. for particles coming from all directions. The number $r_{e}$ measuring the energy of a particle in störmers is connected with the ratio of momentum to charge $(m v / e)$ by Störmer's well-known relation

$$
r_{e}=\sqrt{\frac{m v}{e M_{e}}} R_{e}
$$

where $M_{e}$ is the earth's magnetic moment and $R_{e}$ the earth's radius. Introducing a parameter $r_{\delta}$ similar to $r_{e}$, but referred now to the sun's magnetic dipole moment $M_{s}$ and to the distance from the sun to the earth, $D$, by the relation

$$
r_{s}=\sqrt{\frac{m v}{e M_{s}}} D
$$

the same theory shows that, if the ecliptic lies along the solar magnetic equator, $r_{s}$ must be less than $0 \cdot 414$. Eliminating $m v / e$ from (1) and (2), we obtain :

$$
M_{s}=M_{e}\left(\frac{D}{R_{e}}\right)^{2}\left(\frac{r_{e}}{r_{s}}\right)^{2} ;
$$

which, upon inserting the known magnetic moment of the earth and the known ratio $D / R_{e}$, gives $M_{s}=10^{34}$ E.M.U. If the angle between the sun's magnetic moment and its axis of rotation is small, this value is only slightly changed. It corresponds to a field intensity at the sun's equator of 30 gauss, in good agreement with the results obtained by Hale and his collaborators ${ }^{5}$ from a study of the Zeeman effect of solar lines.

It may be readily shown that, while the sun's magnetic field may thus prevent cosmic particles of energy around $200 \mathrm{mS}$. $\left(2 \times 10^{9} \mathrm{eV}\right.$. if electrons, $1.6 \times 10^{9} \mathrm{eV}$. if protons) from ever reaching the earth, if they come from distances large compared with the diameter of its orbit, it does not hinder low-energy particles of suitable angular momentum shot off the sun from reaching the earth, provided either that such particles leave the sun at very high latitude or that its magnetic moment is inclined at a high angle (around $45^{\circ}$ ) with respect to the ecliptic. This introduces a difficulty in connexion with Birkeland's and Störmer's theory of the aurora polaris, particularly if the particles responsible for the aurora originate in the sunspot belt.

If the interpretation suggested here is correct, there are several consequences which may be accessible to experiment, of which we shall name only two. First there should be a seasonal variation of the intensity of cosmic radiation of lowest energy, of the order of magnitude of the eccentricity of the earth's orbit, with maximum in summer and minimum in winter, arising from the fact that as the earth rotates around the sun the distance between them varies periodically. Secondly, there should be a diurnal variation as a function of solar time with phase which is now being calculated. It arises because the sun's field at the earth is still capable of deflecting particles of energy up to $435 \mathrm{mS}$. approximately, and therefore there is at the earth both a solar allowed cone and a terrestrial allowed cone, the common region of which varies periodically as the earth rotates around its axis. The magnitude of this fluctuation may be roughly estimated at between 0.1 and 0.2 per cent.

It may be added that the possibility of ascribing the blocking of cosmic particles to a magnetic action of the moon seems excluded on the ground that it would have to possess a magnetic moment about ten thousand times that of the earth; this would give rise to large variations of intensity depending on the position of the moon which have not been found.

\section{S. Vallatita}

Massachusetts Institute of Technology, Cambridge, Mass. March 22.

${ }^{1}$ Compton, A. H., Phys. Rev., 43, 398 (1933).

${ }^{2}$ Cosyns, M., Nature, 137, 616 (1936).

3 Janossy, L., Z. Phys., 104, 430 (1937)

4 Lemaître, G., and Vallarta, M. S., Phys, Rev., 50, 493 (1936). For the specification of the energy in störmers, see Phys. Rev., 49, 720 (1936), and for the equivalence between störmers and electronvolts, see Phys. Rev., 43, 90 (1933)

${ }^{5}$ Hale, G. E., Seares, F. H., van Maanen, A., Ellerman, F., Astrophys. J., 47, 206 (1918). 